\title{
Hipersensibilidad dentinaria: un desafío en la práctica odontológica
}

\author{
Dentin hypersensitivity: a challenge in dental practice
}

Fiorela Isabel Mogrovejo Angulo ${ }^{1 a}$

Gabriela Mariana Castro Núñez ${ }^{2 b}$

Wilfredo Gustavo Escalante Otárola ${ }^{1 \mathrm{~b}}$
(iD) https://orcid.org/0000-0002-0762-1067

(iD $h$ ttps://orcid.org/0000-0002-2586-541X

(iD) https://orcid.org/0000-0003-4879-3938

Correspondencia: wilfredoescalante@gmail.com

\section{Resumen}

La hipersensibilidad dentinaria se define como un dolor corto y agudo de la dentina expuesta como reacción a estímulos térmicos, evaporativos, táctiles, osmóticos o químicos. Esta condición normalmente está asociada a lesiones cervicales no cariosas (erosión, abrasión y abfracción), recesiones gingivales, enfermedad periodontal, entre otros problemas odontológicos. Por su naturaleza multifactorial, el diagnóstico de la hipersensibilidad dentinaria suele ser difícil y confuso para el cirujano dentista. No existe una estrategia única que pueda resolver esta condición en todos los pacientes y es de responsabilidad del cirujano dentista conocer sus características para proponer un plan de tratamiento eficaz para sus pacientes. Por tal motivo, la presente revisión tiene como objetivo mostrar las principales características de la hipersensibilidad dentinaria, así como los desafíos asociados a su manejo clínico, para su entendimiento y dominio en la práctica clínica.

Palabras clave: hipersensibilidad de la dentina, odontología general, erosión dental, desmineralización dental

\begin{abstract}
Dentine hypersensitivity is defined as short, sharp pain from exposed dentin in reaction to thermal, evaporative, tactile, osmotic, or chemical stimuli. This condition is associated with non-carious cervical lesions (erosion, abrasion and abfraction), gingival recessions, periodontal disease, among other dental problems. Due to its multifactorial nature, the diagnosis of dentin hypersensitivity is often difficult and confusing for the dental surgeon. There is no single strategy that can solve this condition in all patients and it is the responsibility of the dental surgeon to know its characteristics in order to propose an effective treatment plan for patients. Therefore, this review aims to show the main characteristics of dentin hypersensitivity, as well as the challenges associated with clinical management, for understanding and mastery in clinical practice.

Keywords: dentin hypersensitivity, general practice dental, tooth erosion, tooth demineralization

\footnotetext{
${ }^{1}$ Universidad Nacional Jorge Basadre Grohmann. Facultad de Ciencias de la Salud. Escuela Profesional de Odontología. Semillero de Investigación e Innovación LCNC. Tacna, Perú

${ }^{2}$ Universidad Nacional Mayor de San Marcos. Facultad de Odontología. Departamento Académico de Estomatología Rehabilitadora. Lima, Perú

${ }^{a}$ Estudiante

${ }^{\mathrm{b}}$ Doctor en Odontología. Investigador RENACYT
} 


\section{Introducción}

La hipersensibilidad dentinaria (HD) es una condición clínica cada vez más persistente y problemática que suele pasar desapercibida por los cirujanos dentistas o confundida con otro problema odontológico y que, muchas veces, representa un gran desafío en la práctica clínica. ${ }^{1}$ La HD se puede definir como un dolor corto y agudo de la dentina expuesta como reacción a estímulos térmicos, evaporativos, táctiles, osmóticos o químicos, y que no está relacionado con otro defecto o patología dental. ${ }^{1}$

A pesar de ser un tema ampliamente estudiado, sigue siendo un problema común en adultos, con una prevalencia que varía del $3 \%$ al $98 \%$, entre poblaciones de diferentes características. ${ }^{2}$ Recientemente, Zeola et al. ${ }^{3}$ describieron una prevalencia entre el $11 \%$ y $33 \%$. Por otro lado, diversos estudios han demostrado que la perspectiva del paciente sobre esta condición es diferente a la perspectiva del cirujano dentista. ${ }^{2}$ Según Orchardson y Gillam, ${ }^{4}$ los pacientes que se quejan de los síntomas clásicos de HD, que son de inicio rápido, de carácter agudo y de corta duración, generalmente tienen valores de prevalencia más bajos (15-30 \%) después de un examen clínico. ${ }^{4}$ Por otro lado, en estudios de cuestionarios, donde se depende de la percepción de los pacientes, la HD se podría sobreestimar $(74 \%)^{5,6}$

Otro tema que se ha abordado recientemente en la literatura es el alcance del impacto de la HD en la calidad de vida de las personas que padecen de esta condición. Gibson et al. ${ }^{7}$ observaron que casi el $30 \%$ de los pacientes no pudieron beber agua fría ni ingerir alimentos helados sin referir molestias, así como molestias en su cepillado dental. Si bien varios estudios han sugerido que la HD puede ser un problema importante en algunos pacientes, parece ser un problema relativamente menor para la mayoría de la población debido a su naturaleza transitoria. ${ }^{8}$ Esta situación puede ser una de las razones por las cuales algunos pacientes no informan de este problema al cirujano dentista y tampoco buscan algún tratamiento casero. ${ }^{9,10}$ Así mismo, puede ser una razón por la que los cirujanos dentistas a menudo no diagnostican la HD, a no ser que los pacientes refieran alguna queja. ${ }^{10}$

La HD representa una de las condiciones odontológicas más asociadas con el dolor y de menor éxito en su tratamiento, ${ }^{11}$ justificando así un análisis detallado de las diferentes estrategias de tratamiento disponibles. Por tal motivo, el objetivo de esta revisión de literatura es actualizar a los cirujanos dentistas sobre las principales características de la HD, así como los desafíos asociados a su manejo clínico para su entendimiento y dominio en la práctica clínica. ${ }^{1}$

\section{Mecanismos asociados a la hipersensibilidad dentinaria}

Se han propuesto varias teorías para explicar el mecanismo de la HD, siendo la teoría hidrodinámica, propuesta por Brännström, ${ }^{12}$ la más aceptada. Según esta teoría, los estímulos externos (térmicos, mecánicos, evaporativos y osmóticos) conducen al movimiento de líquido dentro de los túbulos dentinarios y este movimiento estimula indirectamente los extremos del nervio pulpar, causando una sensación dolorosa. ${ }^{12}$

En términos generales, la HD se diferencia de otros dolores de dientes, porque está asociada a las fibras $A \delta$; estimuladas, principalmente, por la aplicación de un estímulo frío que produce un dolor agudo (que se atenúa al ser retirado el estímulo) en comparación con la estimulación de las fibras $\mathrm{C}$, que producen un dolor sordo. ${ }^{13}$

Para fines prácticos, la teoría hidrodinámica promueve dos enfoques básicos para el tratamiento de la dentina hipersensible:

1) Obliterando túbulos dentinarios abiertos, localizados en regiones de dentina expuesta originada por pérdida de esmalte o por una recesión gingival, con permeabilidad tanto para la 
cavidad oral como para la pulpa. ${ }^{6}$ Reduciendo así cualquier movimiento de fluido provocado por el estímulo y, consecuentemente, evitando la transmisión del estímulo externo a la pulpa. ${ }^{14}$

\section{Figura 1}

Imagen microscópica de la obliteración de túbulos dentinarios, luego de la aplicación de un agente remineralizante a base de fosfato de calcio nanoparticulado
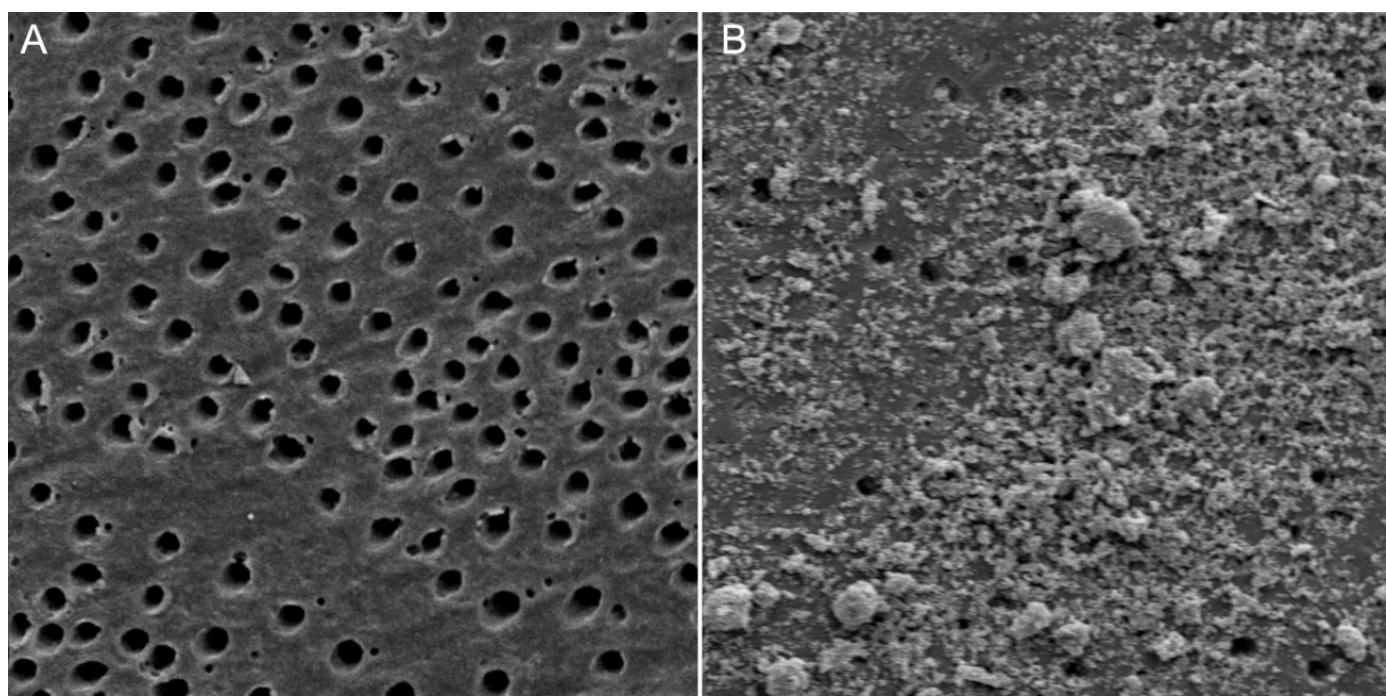

A. Túbulos dentinarios abiertos en dentina erosionada. B. Obliteración de túbulos dentinarios después de tratamiento con Desensibilize Nano P (FGM) a base de nanopartículas de fosfato de calcio.

2) Reduciendo la excitabilidad nerviosa en el interior del túbulo dentinario, mediante la difusión de sales de potasio, despolarizando el potencial eléctrico de las células e inhibiendo la respuesta nerviosa por los movimientos del líquido intratubular. ${ }^{14,15}$

\section{Etiología de la hipersensibilidad dentinaria y factores predisponentes}

Se han implicado varios factores etiológicos y predisponentes en el inicio de la HD, como son la abrasión, abfracción, erosión, recesión gingival, calidad del hueso alveolar, enfermedad periodontal y su tratamiento, procedimientos quirúrgicos y restauradores, entre otros. ${ }^{16}$ En la tabla 1 se presentan los principales factores etiológicos y predisponentes asociados a HD.

Tabla 1

Factores etiológicos y predisponentes asociados con hipersensibilidad dentinaria

- Pérdida de esmalte

- Denudación de cemento

- Recesión gingival

- Desgaste

- Abrasión

- Abfracción

- Erosión (intrínseca y extrínseca)

- Malposición dental

- Adelgazamiento, fenestración y ausencia de hueso alveolar vestibular

- Enfermedad periodontal y su tratamiento

- Cirugía periodontal, tratamiento restaurador

- Hábitos del paciente

Fuente: adaptado de Chabanaki y Gillam ${ }^{17}$ 
Más recientemente, varios investigadores han sugerido que la HD puede ser un fenómeno de desgaste dental caracterizado principalmente por erosión química diaria y recurrente, que con el tiempo puede exponer la superficie de la dentina e iniciar las lesiones del desgaste dental. ${ }^{17-19}$ Dababneh et al. ${ }^{20}$ sugirieron dos procesos biológicos específicos asociados con los factores etiológicos mencionados anteriormente, asociados a la HD:

1) Localización de la lesión. La dentina queda expuesta como resultado de la pérdida de esmalte y/o pérdida de tejidos blandos asociada con la recesión gingival (incluida la pérdida de cemento). ${ }^{20}$

2) Inicio de la lesión. Una vez que la dentina ha sido expuesta, los túbulos dentinarios permeables se abrirán al entorno de la cavidad oral y de esta manera, cualquier estímulo posterior (como el frío) iniciará el movimiento del líquido intratubular, activando a los mecanoreceptores del complejo dentino/pulpar. ${ }^{20}$

La literatura tradicional afirma que la HD se produce como resultado de un control de placa exhaustivo en una boca sana y que afecta principalmente a las superficies vestibulares de los dientes; ${ }^{5}$ sin embargo, se conoce que esta condición también puede estar asociada a enfermedad periodontal y/o su tratamiento. ${ }^{5}$

Recientemente, el término 'sensibilidad radicular' (SR) fue introducido por la Federación Europea de Periodoncia para describir la sensibilidad dental asociada con enfermedad periodontal y/o terapia periodontal, ${ }^{21}$ en contraste con el punto de vista tradicional de HD asociada a individuos con buen control de la placa. ${ }^{5}$ Actualmente, no parece haber ninguna evidencia sustancial de datos de prevalencia (en la literatura publicada) para distinguir entre estas dos condiciones. ${ }^{4}$

\section{Características clínicas de la hipersensibilidad dentinaria}

La evidencia científica actual sugiere que los dientes más comúnmente asociados con HD son los caninos, premolares y molares. ${ }^{7}$ En términos generales, la cara vestibular de estos dientes se expone con mayor frecuencia, probablemente como resultado de un cepillado excesivo y/o incorrecto, en asociación con otros factores etiológicos; ${ }^{5,9}$ sin embargo, puede haber diferentes factores predisponentes asociados con la HD y estas características deben considerarse cuidadosamente al decidir una estrategia para su tratamiento. La tabla 2 muestra diferentes estrategias recomendadas para el manejo de la hipersensibilidad dentinaria. manera:

De acuerdo con Gillam et al., ${ }^{9}$ los pacientes con HD pueden clasificarse de la siguiente

- Aquellos que tienen bocas relativamente sanas e HD como resultado de una higiene oral meticulosa y quizá demasiado intensa;

- Aquellos que se quejan de HD como resultado de una enfermedad periodontal, y/o su tratamiento, y también pueden tener preocupaciones estéticas relacionadas con la pérdida de tejido gingival (recesión gingival);

- Aquellos que se quejan de HD como resultado de otros problemas con los dientes. 
Tabla 2

Estrategia de manejo de la hipersensibilidad dentinaria

RECESIÓN GINGIVAL
Evaluación clínica
- Medir clínicamente el
defecto de recesión gingival.
- Tomar modelos de estudio
y fotografías clínicas para
controlar la condición a lo
largo del tiempo.
- Verificar y controlar la salud
periodontal.
- Identificar y corregir de
factores predisponentes o
precipitantes.
- Usar escalas de dolor para
evaluar y monitorear la HD.

\section{Educación del paciente}

- Mostrar al paciente los sitios afectados.

- Explicar la causa probable de la recesión.

- Explicar los factores que desencadenan episodios de dientes sensibles.

- Alentar a los pacientes a modificar su régimen de higiene bucal para reducir el daño a las encías.

- Aconsejar la reducción del consumo excesivo de alimentos y bebidas ácidas.

Opciones correctivas
-Tratamiento de ortodoncia.
-Restauración del tejido
desgastado y márgenes
subgingivales.
- Obturación de túbulos
dentinarios con láser.
- Uso de agentes
desensibilizantes.
-Para defectos de recesión
gingival localizada, pueden
considerarse procedimientos
quirúrgicos de injerto de
tejido blando (cobertura de la
raíz).
- Extirpación pulpar (casos
extremos).

\section{DESGASTE DENTAL}

Evaluación clínica

- Identificar la causa del desgaste dental.

- Registrar la gravedad de las lesiones, si es posible, utilizando un índice reconocido.

- Tomar modelos de estudio y fotografías clínicas para controlar la condición a lo largo del tiempo.

- Verificar y controlar la salud periodontal.

- Usar escalas de dolor para evaluar y monitorear la HD.

\section{Educación del paciente}

- Mostrar al paciente los sitios y explicar la causa probable de las lesiones en los dientes.

- Recomendar un régimen de higiene oral para minimizar el riesgo de otros dientes.

- Recomendar reducir la frecuencia de consumo de alimentos y bebidas ácidas.

\section{Opciones correctivas}

- Proporcionar tratamiento remineralizante con alto contenido de flúor (fase preventiva).

- Proporcionar tratamiento profesional desensibilización para aliviar la HD.

- Alentar al paciente a una interconsulta médica, si el desgaste es causado por el ambiente de trabajo o por reflujo/vómitos excesivos.

- Corrección restauradora con resinas compuestas; las coronas también pueden ser apropiadas.
TRATAMIENTO PERIODONTAL

\section{Evaluación clínica}

- Identificar la enfermedad periodontal 0 tratamiento periodontal como la causa principal de exposición de la dentina y la HD asociada.

- Verificar y controlar la salud periodontal.

- Usar escalas de dolor para evaluar y monitorear la HD.

\section{Educación del paciente}

- Reforzar la necesidad de una buena higiene bucal.

- Mostrar al paciente los sitios afectados por la enfermedad periodontal $y$ explicar la causa probable de la dentina expuesta.

- Guiar al paciente para mejorar el régimen de higiene bucal 'en casa'.

- Instruir sobre medidas para reducir los factores de riesgo periodontal.

\section{Tratamiento}

\section{Fase inicial}

-Procedimientos

periodontales no quirúrgicos.

- Tratamiento de la HD.

\section{Reevaluación}

-Evaluación de seguimiento del estado periodontal y de la HD.

\section{Fase correctiva}

-Procedimiento periodontal quirúrgico.

- Tratamiento de la HD.

Fase de mantenimiento

- Terapia periodontal de apoyo.

- Monitoreo continuo de la salud periodontal.

- Tratamiento de la HD.

- Consejos de higiene oral.

Fuente: Gillam et al. ${ }^{9}$ (modificado) 
Por lo tanto, estas pautas propuestas fueron diseñadas para ayudar al cirujano dentista a abordar las diferentes características asociadas con HD, con un enfoque más personalizado, en lugar de simplemente seguir una estrategia de gestión generalizada. ${ }^{9}$

\section{Diagnóstico de hipersensibilidad dentinaria}

El diagnóstico clínico del dolor orofacial puede ser un procedimiento difícil y que requiere mucho tiempo, especialmente en un entorno limitado de la práctica clínica. ${ }^{5}$ El diagnóstico de pacientes con HD puede ser problemático por varias razones; en primer lugar, la dificultad para identificar áreas de la boca que pueden estar causando el problema y, en segundo lugar, la naturaleza altamente subjetiva del dolor y su variabilidad entre pacientes.

Es importante recordar que la definición de HD es, esencialmente, un diagnóstico de exclusión y esto debe motivar al cirujano dentista a excluir cualquier otra condición que interfiera en el diagnóstico definitivo. ${ }^{4}$ Por lo tanto, es esencial que el cirujano dentista obtenga un historial médico y odontológico completo de los pacientes, ${ }^{4}$ así como el historial del dolor (sitio, inicio, síntomas asociados, atenuantes, duración y frecuencia, factores de exacerbación, gravedad, etc.). ${ }^{5}$

En la práctica clínica, el uso de una sonda exploradora y un chorro de aire de una jeringa triple, como estímulos para causar la incomodidad del paciente por HD, son aceptables para la identificación de sitios susceptibles y para clasificar la severidad del dolor. ${ }^{17}$ Adicionalmente, el uso de una escala de puntuación numérica simple, como una escala visual analógica del 0 al 10, es apropiada para registrar la percepción de HD del paciente en la primera cita y poder monitorear la evolución de su dolor en las consultas posteriores. ${ }^{14}$

\section{Manejo clínico de la hipersensibilidad dentinaria}

Ha habido una serie de paradigmas sobre los tratamientos recomendados para el manejo de la $\mathrm{HD},{ }^{4,10,22}$ pero aún existen dudas sobre si estas medidas pueden llevarse a cabo efectivamente en la práctica clínica. Actualmente, en pacientes con recesiones gingivales es recomendable la toma de modelos de estudios y fotografías clínicas para monitorear esta condición y el estado periodontal a lo largo del tiempo. ${ }^{7}$

También es importante identificar y corregir cualquier factor predisponente o precipitante que pueda ser responsable del desarrollo de la recesión gingival; por ejemplo, hábitos del paciente, técnica incorrecta de cepillado dental, etc. ${ }^{17}$ Estos factores pueden discutirse con el paciente para evitar cualquier daño adicional a los tejidos duros y blandos que puedan aumentar la gravedad del dolor asociado con la HD, junto con una recomendación de evitar el consumo excesivo de alimentos y bebidas ácidas. ${ }^{13}$

Se han propuesto varios materiales y protocolos de tratamiento basados en nitrato de potasio, cloruro de estroncio y, recientemente, nuevos compuestos de fosfato de calcio; sin embargo, aún existen muchas dudas en relación con los diferentes protocolos de desensibilización y sobre el mejor agente que oblitera los túbulos dentinarios y protege la dentina expuesta en presencia de ácidos intrínsecos y desafíos extrínsecos. ${ }^{23,24}$ Productos a base de fosfato de calcio muestran resultados positivos para el tratamiento de hipersensibilidad dentinaria severa, hasta conseguir la obliteración de túbulos dentinarios y remineralización de dentina expuesta. ${ }^{25}$ 


\section{Figura 2}

Protocolo de aplicación de un producto a base de fosfato de calcio nanoparticulado
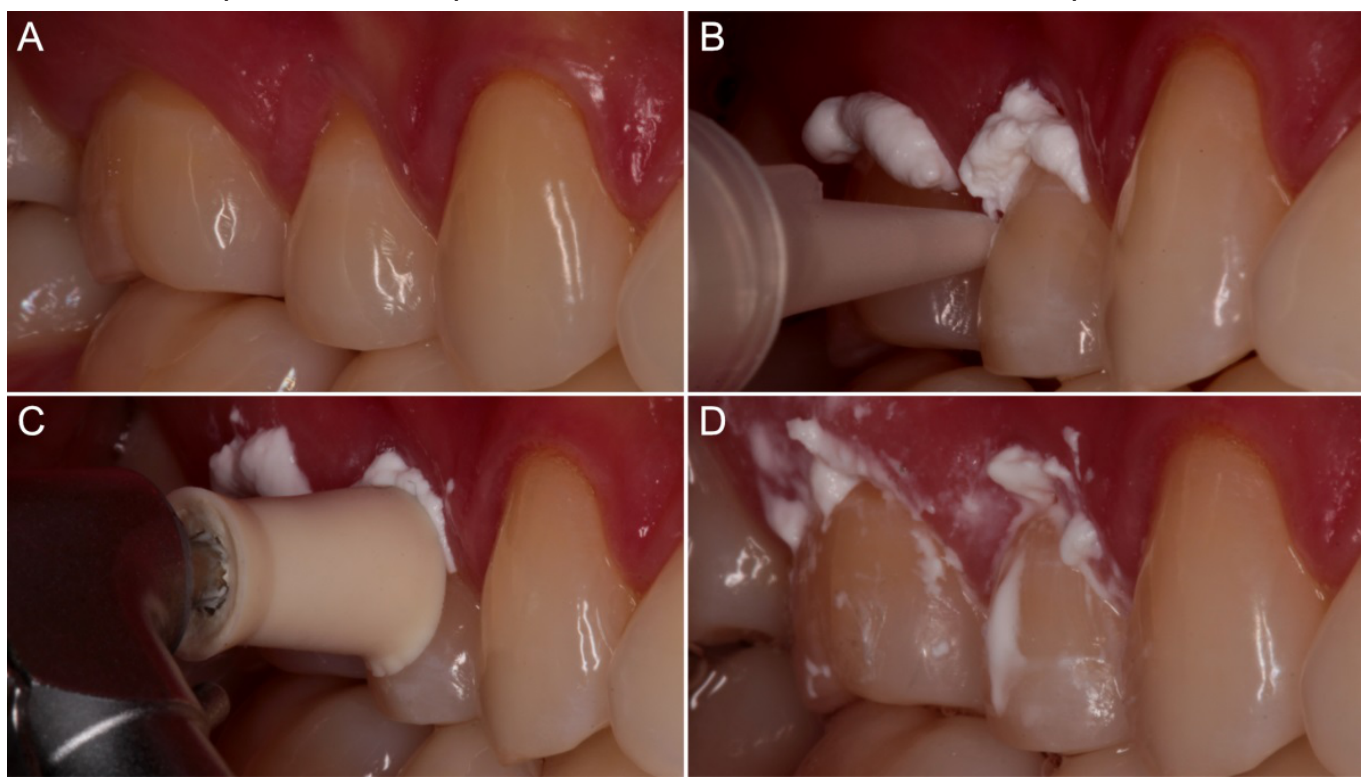

A. Elementos 25 y 26 con recesiones gingivales e hipersensibilidad severa. B. Aplicación de pasta Desensibilize Nano P (FGM) sobre dentina expuesta. C. Fricción con copa de goma a baja velocidad por 10 segundos. D. Reposo por 5 minutos.

Dependiendo de la gravedad, el cirujano dentista también puede recomendar el uso de pastas de dientes o enjuagues bucales desensibilizantes para uso doméstico. ${ }^{17}$ También es esencial contar con una estrategia de monitoreo basada en la gravedad de la afección. Pacientes que han sido diagnosticados con HD asociada con problemas de desgaste dental o enfermedad periodontal también pueden manejarse con una estrategia similar, específicamente basada en sus características clínicas (Tabla 2). Por lo tanto, el manejo exitoso de la HD, no solo implica el diagnóstico correcto de la afección por parte del cirujano dentista, sino que también incluye la importancia de implementar estrategias de prevención que eliminen o limiten cualquier deterioro adicional mediante tratamientos apropiados, consejos dietéticos y monitoreo de la condición. ${ }^{4,9}$

\section{Conclusiones}

La información contenida en esta revisión de literatura proporciona una guía práctica basada en la evidencia científica actual sobre el manejo de la hipersensibilidad dentinaria desde la prevención, diagnóstico, monitoreo y tratamiento. No existe una estrategia única que pueda resolver esta condición en todos los pacientes, por tal motivo, es de responsabilidad del cirujano dentista conocer sus características y tener la capacidad de proponer un plan de tratamiento eficaz para sus pacientes.

\section{Referencias}

1. Holland GR, Narhi MN, Addy M, Gangarosa L, Orchardson R. Guidelines for the design and conduct of clinical trials on dentine hypersensitivity. Journal of clinical periodontology. 1997;24(11):808-13.

2. Splieth $\mathrm{CH}$, Tachou A. Epidemiology of dentin hypersensitivity. Clinical oral investigations. 2013;17 Suppl 1:S3-8.

3. Favaro Zeola L, Soares PV, Cunha-Cruz J. Prevalence of dentin hypersensitivity: Systematic review and meta-analysis. Journal of dentistry. 2019;81:1-6. 
4. Orchardson R, Gillam DG. Managing dentin hypersensitivity. Journal of the American Dental Association. 2006;137(7):990-8; quiz 1028-9.

5. Gillam DG, Orchardson R. Advances in the treatment of root dentine sensitivity: mechanisms and treatment principles. Endodontic Topics. 2006;13(1):13-33.

6. Cunha-Cruz J, Stout JR, Heaton LJ, Wataha JC, Northwest P. Dentin hypersensitivity and oxalates: a systematic review. Journal of dental research. 2011;90(3):304-10.

7. Gibson B, Boiko OV, Baker S, Robinson PG, Barlow A, Player T, et al. The impact of dentine sensitivity: personal and fuctional aspects. Soc Sci Dent. 2010;1:11-20.

8. Gillam DG. Current diagnosis of dentin hypersensitivity in the dental office: an overview. Clinical oral investigations. 2013;17 Suppl 1:S21-9.

9. Gillam D, Chesters R, Attrill D, Brunton P, Slater M, Strand P, et al. Dentine hypersensitivity-guidelines for the management of a common oral health problem. Dental update. 2013;40(7):514-6, 8-20, 23-4.

10. Canadian Advisory Board on Dentin H. Consensus-based recommendations for the diagnosis and management of dentin hypersensitivity. Journal. 2003;69(4):221-6.

11. Idon PI, Esan TA, Bamise CT. Efficacy of Three In-Office Dentin Hypersensitivity Treatments. Oral health \& preventive dentistry. 2017;15(3):207-14.

12. Brannstrom M. Dentin sensitivity and aspiration of odontoblasts. Journal of the American Dental Association. 1963;66:366-70.

13. Narhi M, Kontturi-Narhi V, Hirvonen T, Ngassapa D. Neurophysiological mechanisms of dentin hypersensitivity. Proceedings of the Finnish Dental Society Suomen Hammaslaakariseuran toimituksia. 1992;88 Suppl 1:15-22.

14. Gillam DG. Management of fentin hypersensitivity. Curr Oral Health Resp. 2015;2:87-94.

15. Brannstrom $M$, Astrom $A$. The hydrodynamics of the dentine; its possible relationship to dentinal pain. International dental journal. 1972;22(2):219-27.

16. Chabanski MB, Gillam DG. Aetiology, prevalence and clinical features of cervical dentine sensitivity. Journal of oral rehabilitation. 1997;24(1):15-9.

17. West NX, Sanz M, Lussi A, Bartlett D, Bouchard P, Bourgeois D. Prevalence of dentine hypersensitivity and study of associated factors: a European population-based crosssectional study. Journal of dentistry. 2013;41(10):841-51.

18. Bartlett DW, Lussi A, West NX, Bouchard P, Sanz M, Bourgeois D. Prevalence of tooth wear on buccal and lingual surfaces and possible risk factors in young European adults. Journal of dentistry. 2013;41(11):1007-13.

19. Olley RC, Moazzez R, Bartlett D. The relationship between incisal/occlusal wear, dentine hypersensitivity and time after the last acid exposure in vivo. Journal of dentistry. 2015;43(2):248-52.

20. Dababneh RH, Khouri AT, Addy M. Dentine hypersensitivity - an enigma? A review of terminology, mechanisms, aetiology and management. British dental journal. 1999;187(11):606-11; discussion 3.

21. Sanz M, Addy M. Group D summary. Journal of clinical periodontology. 2002;29(3):195-6.

22. Addy $M$, Urquhart E. Dentine hypersensitivity: its prevalence, aetiology and clinical management. Dental update. 1992;19(10):407-8, 10-2.

23. Escalante-Otarola WG, Castro-Nunez GM, Jordao-Basso KCF, Guimaraes BM, Palma-Dibb RG, Kuga MC. Evaluation of dentin desensitization protocols on the dentinal surface and their effects on the dentin bond interface. Journal of dentistry. 2018;75:98-104.

24. Alencar CM, de Paula BFL, Ortiz MIG, Magno MB, Silva CM, Maia LC. Clinical efficacy of nano-hydroxyapatite in dentin hypersensitivity: A systematic review and meta-analysis. Journal of dentistry. 2019;82:11-21.

25. Escalante-Otárola W, Castro-Núñez G, Jordao-Basso KCF, Lima SL, Kuga MC, Bandeca MC. Treatment Protocol for Dentin Hypersensitivity. World Journal of Dentistry. 2017;8(1):1-4. 